\title{
Potential of Impedance based Battery Diagnosis for Electro Mobility
}

\author{
Kanoun, O. Tröltzsch, U. Büschel, P. \\ Technische Universität Chemnitz \\ Professur für Mess- und Sensortechnik \\ Reichenhainer Straße 70 \\ 09126 Chemnitz
}

\begin{abstract}
The rapid adoption of electric vehicles in the near future will require significant efforts in the field of battery technology. An integral part of this is to provide a reliable diagnosis for a variety of information required. Impedance spectroscopy is one suitable and efficient method to obtain the required information. It provides information on the condition of the battery that can be used in the energy management of the vehicle as well as for a state monitor during the whole life cycle of the battery. Determination of state of charge or battery capacity can be supported by impedance spectroscopy. The driving range of the vehicle depending on load conditions can be determined by time domain simulations. The use of the battery can be controlled while simultaneously minimizing aging. Various models for batteries can be used, for example, on the basis of ordinary, nonlinear differential equations, digital filters or equivalent circuits together with the corresponding methods for system identification in the time or frequency domain. Fast measurement methods of impedance spectra and stochastic methods for nonlinear parameter optimization are supporting an efficient and reliable information retrieval. Thereby a significant advantage over the prior art in terms of shorter measuring and computing time and higher reliability of the algorithms can be achieved.
\end{abstract}

\section{Introduction}

Right now there is the consensus in politics and society to rapidly change the energy source of our daily mobility from a fossile to a regenerative based one. The use of regenerative power sources requires powerfull energy storage devices. Most of these devices will be electrochemical based like batteries and double layer capacitors (DLC). Due to the high costs of batteries for electric and hybride vehicles a powerful diagnosis is essential to protect the battery and to ensure long lifetime and high reliability.

Various diagnostic methods exist which can be separated into invasive and non-invasive methods. For the diagnosis of vehicle batteries only non-invasive methods are applicable. These non-invasive methods involve static discharge tests, pulse pattern tests [1,2], methods that perform an online estimation with Kalman filters [3], state estimation with neural networks [4], unsharp classification [5], electrochemical impedance spectroscopy (EIS) [6,7] and others. Among these methods EIS can be used in all steps of the life cycle of a battery, ranging from product development, quality assurance and characterization tests to in-application diagnostics. EIS can give insight into electrochemical processes inside the battery thus helping to optimize its usage regarding aging and power handling capability.

In the vehicle the information gained by EIS can be used to determine the state of charge and aging influences [7] or to simulate the battery response for a given load [8]. In a complex system like an electric vehicle this helps to estimate the possible range with the remaining charge and to optimize the power management strategy. With a more comprehensive view, the detailed information about the battery can be used for an optimized integration of vehicles into a smart grid and for a wear leveling of a certain number of batteries.

\section{Battery models and system identification}

Battery models are essential for battery simulation and battery diagnosis. For battery diagnosis they provide the opportunity to compact information obtained from impedance measurements. For time domain simulations models are required as underlying simulation equations. For determining the 
parameters of the models, measured data is required that can be obtained in the frequency and time domain. Both of them have different advantages. Time domain data is very easy to acquire but it is more complex, to determine model parameters directly from time domain data without any previous signal processing. Frequency domain data, for example spectra of measured currents and voltages or impedance spectra, in some cases allow a direct determination of model parameters such as series or charge transfer resistances. For determining frequency domain data, signal processing is always required for example a fast fourier transform.

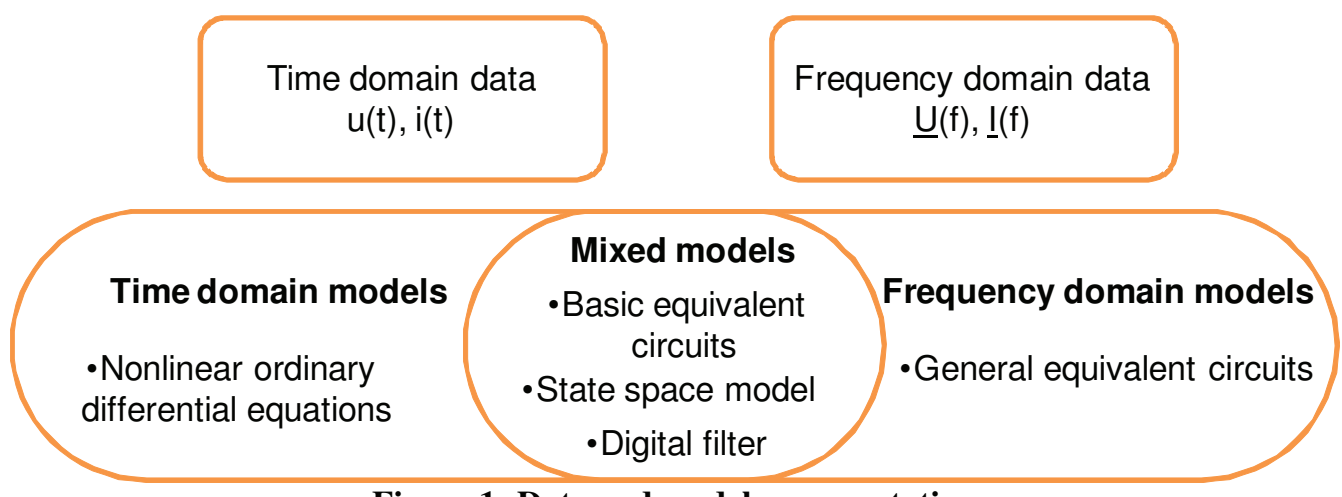

Figure 1: Data and model representation

Similar to measured data, battery models can be derived as time domain models, frequency domain models but also as mixed models. Equivalent circuits are the most popular battery models. Basic equivalent circuits can be considered as mixed models because they have equivalent representations in time and frequency domain that are linked by certain transforms without any loss of information between both domains. State space models and digital filters are very similar to basic equivalent circuits because they also have a transformation that links time and frequency domain representation without loss of any information. In the case of constant phase elements are present in the models, the model is be considered as frequency domain model because it cannot be transformed directly in the time domain [6]. The link between time and frequency domain for constant phase element models can be established by fractional-order control theory and will result in approximate models in the time domain [9]. In the case of considering nonlinearities, such as nonlinear charge transfer reactions, time domain models are more suitable because for example the butler volmer equation can directly be inserted in an ordinary differential equation system [10]. In this case the time domain model cannot be converted into a frequency domain model in general. A transform is only possible for a working point.

For the determination of model parameters linear and nonlinear deterministic and stochastic optimization methods are applied. Linear optimization methods are only applicable to basic linear problems. In the field of battery diagnosis the optimization problems are nonlinear in most cases. Deterministic methods such as the Levenberg Marquardt method are a quasi standard for nonlinear parameter optimization. For an unattended, automated nonlinear parameter optimization, stochastic methods are preferable because residuals for large parameter search spaces are smaller compared to deterministic methods [11].

\section{Applications}

In the following sections some examples applying impedance spectroscopy that are relevant for eclectic mobility are discussed. The examples cover the field of impedance determination, analyzing the aging behavior of a battery and voltage prediction. These fields are important because they support a proper operation of the battery by detecting the actual battery health, minimize battery ageing and maintaining safe operation conditions.

\subsection{Fast impedance measurement}

For the parameterization of battery models in the frequency domain, frequency domain data is required. Data is obtained by impedance measurements. Especially in the field of batteries the frequencies of interest are very low and the time for measurement is long accordingly. To shorten time required for impedance measurement several signals for example rectangular, noise and multi sine impedance measurements can be used. Using multi sine signals is very advantageous because measurement time can be reduced about $50 \%$ and the signal to noise ratio is very good compared to other signals [12]. For calculating the impedance from a multi sine time domain signal, first the signal is multiplied by a window 
function to reduce the leakage effect. After that, zeros are appended to improve the frequency resolution. The result of the fast fourier transform show the spectral lines of the frequencies contained in the multi sine signal. From amplitude and phase of each spectral line the impedance can be calculated for each frequency [13].

1. Test current: time domain

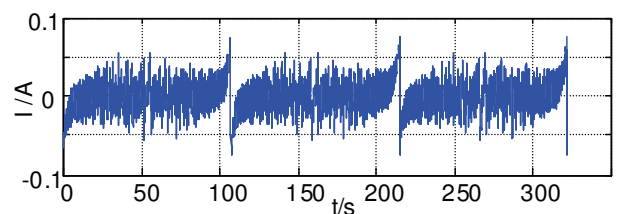

2. Multiplication of window function

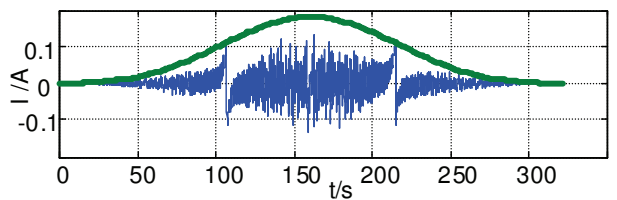

3. Appending zeros for interpolation

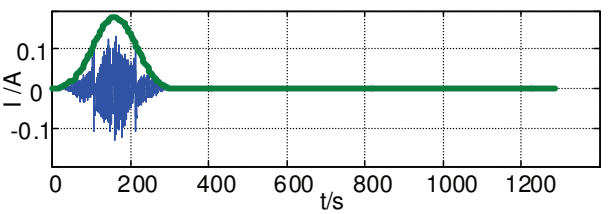

4. Result of Discrete Fourier Transform

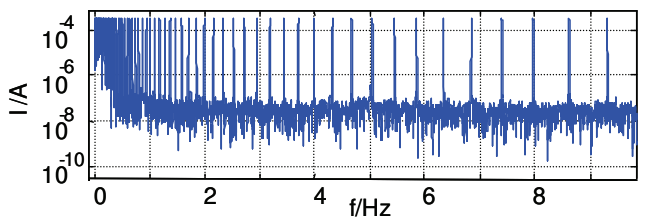

Figure 2: Extracting impedance from multi sine signals

In technical applications related to electric mobility these techniques can be used to faster determine impedance lookup tables for battery behavior at different temperature and state of charge.

\subsection{Analyzing aging behavior}

The aging behavior of a battery can be analyzed by fitting impedance models to measured impedance data. The Randles equivalent circuit, which has been derived for describing electrode kinetics, is a very basic and well understood impedance model [14]. The following equations show the impedance model equation, containing a charge transfer resistance $R_{D}$, a double layer capacitance $C_{D}$ and a Warburg diffusion element $\sigma_{\mathrm{W}}$.

$$
\begin{aligned}
& \underline{Z}=R_{s}+R_{D} \frac{1}{j \frac{\omega}{\omega_{g, C}}+\frac{1}{1+\sqrt{\frac{\omega_{g, \sigma}}{\omega}}(1-j)}} \\
& \omega_{g, C}=\frac{1}{C_{D} R_{D}}, \quad \omega_{g, \sigma}=\left(\frac{\sigma_{W}}{R_{D}}\right)^{2}, k=\frac{\omega_{g, \sigma}}{\omega_{g, C}}
\end{aligned}
$$

The Randles equivalent circuit is normalized in the given equation. Normalization allows a more robust parameter extraction and contributes to a more technical and less chemical interpretation of the fitting results. From measured parameters, threshold values can be determined, defining the end of life of a battery. In the example given in figure 3 the major contribution to aging comes from the charge transfer resistance $R_{D}$. So its value can be used to define an end of life criteria additional to the often used value of $R_{E}$, that contributes less significant to aging in the given example.
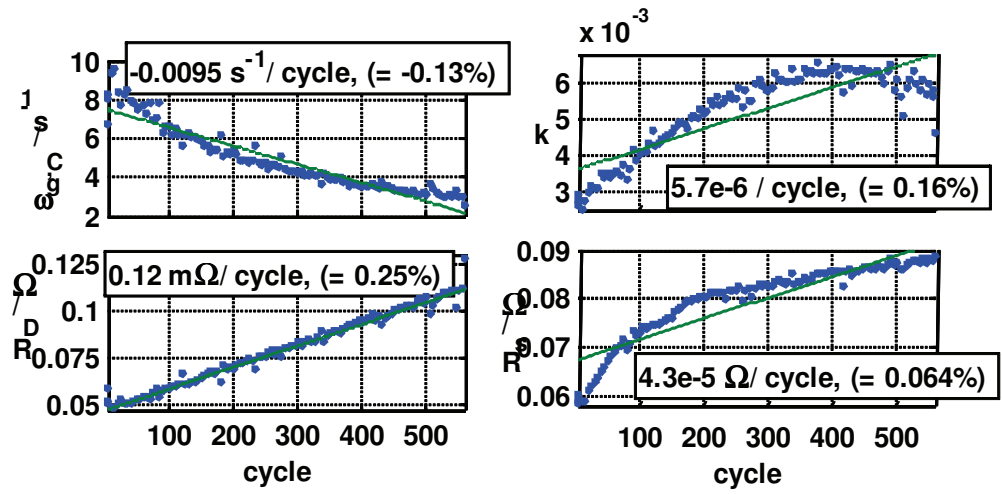

Figure 3: Analysis of aging behavior 


\subsection{Time domain simulation}

Simulation of the battery voltage is a challenging task related to electric mobility. It is required for predicting vehicle range under certain load conditions for example a certain speed or altitude profile. It is also required for predicting the short time behavior of the battery under certain load conditions for example during acceleration or the use of ancillary units. Predicting the battery voltage is based on simulation models and its parameters. For determining model parameters in the frequency and time domain examples will be discussed in the following sections.

\subsubsection{Frequency domain parameter extraction}

Determining model parameters from the frequency domain is very similar to the methodology applicable to analyzing the aging behavior. For simulating the battery behavior in the time domain a simple model considering battery voltage, a series resistance and Warburg diffusion is considered. Figure 4 shows the corresponding equivalent circuit. For the constant phase element used, a suitable time domain representation is required. Therefore several methods for approximating fractional order systems by rational order system are available [15]. The method described in [16] is very convenient because it allows a straight forward digital filter realization [8].

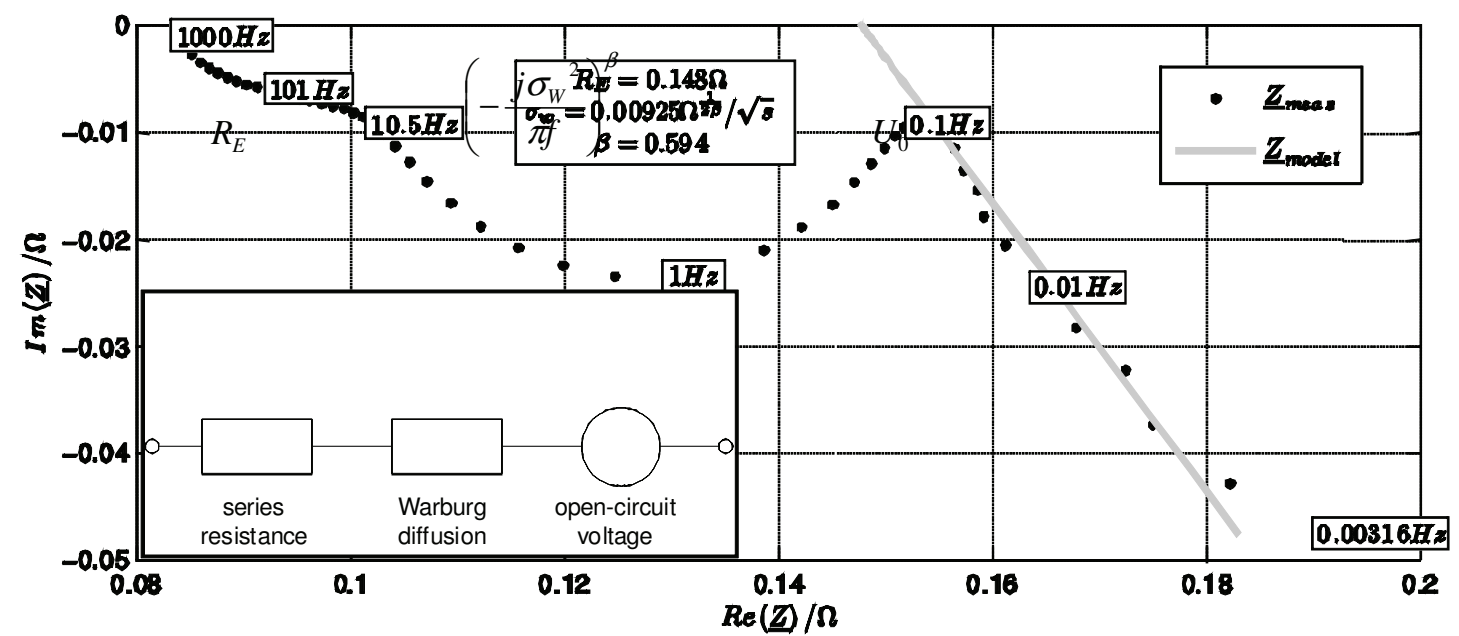

Figure 4: Determinging time domain model parameters from frequency domain data

Figure 5 shows the voltage simulation results. After a simulation time of 1000 s the voltage deviation is less than $1 \mathrm{mV}$. At a time of 600 s there is a small deviation of $20 \mathrm{mV}$ due to the changing state of charge.
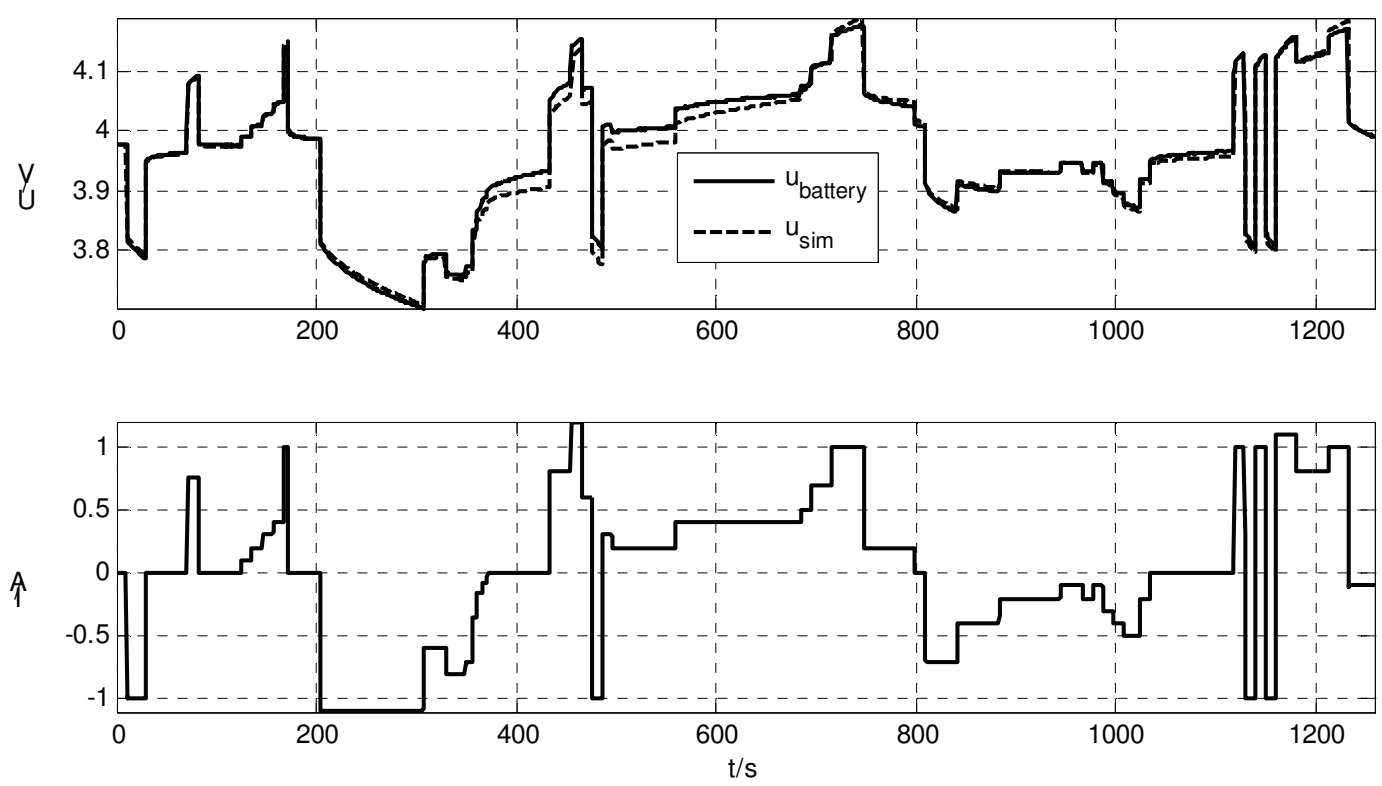

Figure 5: Results of the time domain simulation 
The deviation is caused by the changing battery parameters that are not considered during time domain simulation. The model parameters are kept constant during the whole simulation. By also changing the model parameters during simulation the observed deviation can further be reduced.

\subsubsection{Time domain parameter extraction}

Performing time domain simulations using parameters extracted from time domain data is the most challenging task. It can for example be used to extract battery parameters from regular voltage and current fluctuations that are cause by several electrical loads. Figure 6 shows a measured voltage and current profile of a battery cell that might occur by the operation of a windshield wiper.
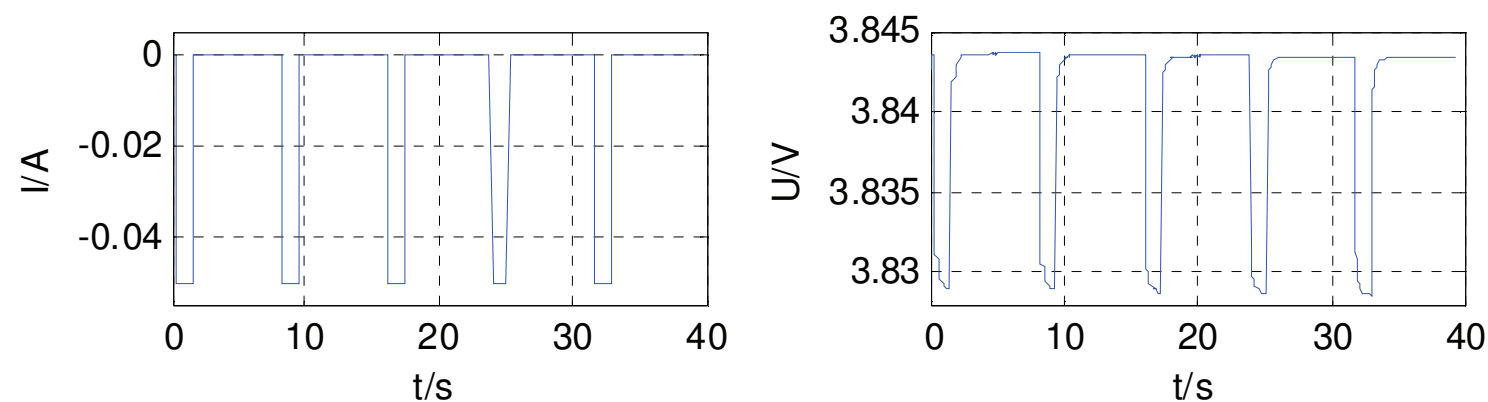

Figure 6: Measured data for model parameterization

From the shown signal, parameters of linear and nonlinear dynamic battery models can be derived and used for the simulation of other loads for example the starter or other ancillary units. Figure 7 shows an example for a starting pulse simulated with the parameters extracted from the windshield wiper data and the corresponding measured battery voltage. From time domain simulation results a decision is possible whether an ancillary unit can be operated or not by not exceeding certain voltage limits.
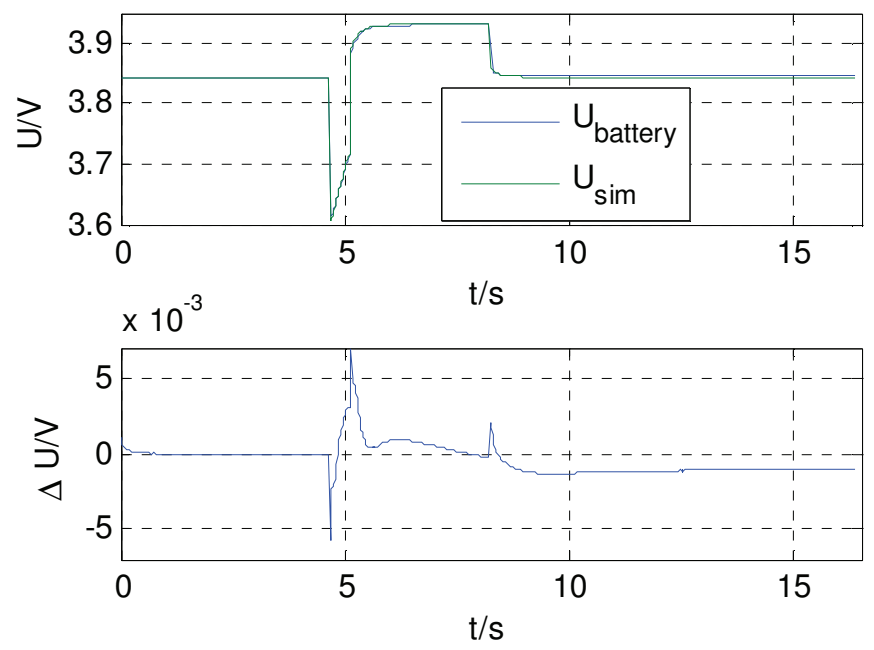

Figure 7: Comparison of measured and simulated voltage

\section{Conclusion}

Many methods based on impedance spectroscopy are applicable to electric mobility. Fast impedance measurements accelerate the data acquisition process. Analyzing the aging behavior using compact impedance models allows defining reliable battery health indicators and end of life criteria. Voltage predictions help to decide on driving profiles, estimate the vehicle range and operating ancillary units. These tasks can be solved by using proper categorizing battery models and measured data in time and frequency domain and clarifying their relations. A scheme of time and frequency domain models and data will also support the identification of solutions for other technical challenges in the field of electric mobility. 


\section{References}

[1] USCAR: USABC Electric Vehicles Test Procedures Manual. USCAR, January 1996

[2] Coleman, M. ; Hurley, W. G. ; Lee, C. K.: An Improved Battery Characterization Method Using a Two-Pulse Load Test. In: IEEE Transactions on Energy Conversation 23 (2008), p. 708-713. - DOI 10.1109/TEC.2007.914329

[3] Plett, Gregory L.: Extended Kalman filtering for battery management systems of LiPB-based HEV battery packs: Part 2. Modeling and identification. In: Journal of Power Sources 134 (2004), No. 2, p. 262-276. - DOI 10.1016/j.jpowsour.2004.02.032

[4] Shen, W. X. ; Chan, C. C. ; Lo, E. W. C. ; Chau, K. T.: A new battery available capacity indicator for electric vehicles using neural network. In: Energy Conversion and Management 43 (2002), p. 817826. - DOI 10.1016/S0196-8904(01)00078-4

[5] Salkind, Alvin J. ; Fennie, Craig ; Singh, Pritpal ; Atwater, Terrill ; Reisner, David E.: Determination of state-of-charge and state-of-health of batteries by fuzzy logic methodology. In: Journal of Power Sources 80 (1999), No. 1-2, p. 293-300. - DOI 10.1016/S0378-7753(99)00079-8

[6] Barsoukov, Evgenij (Hrsg.) ; MacDonald, J. R. (Hrsg.): Impedance Spectroscopy. 2nd edition. Hoboken, New Jersey : Wiley-Interscience, 2005

[7] Tröltzsch, U.: Modellbasierte Zustandsdiagnose von Gerätebatterien. Düsseldorf : VDI Verlag, 2006 (Fortschritt-Berichte Reihe 8 Nr. 1088, Dissertation)

[8] Tröltzsch, U. ; Büschel, P. ; Kanoun, O.: Time domain simulations of constant phase elements using IIR filter. In: International Workshop on Impedance Spectroscopy. Chemnitz, Deutschland, 13.-15. Oktober 2010

[9] Podlubny, Ignor; Thimann, Kenneth V. : Fractional Differential Equations: An Introduction to Fractional Derivatives, Fractional Differential Equations, to Methods of Their Solution and Some of Their Applications. Bd. 198. Elsevier, 1998

[10] Brad, Allen J.; Faulkner, Larry R.: Electrochemical Methods. 2nd edition. New York : John Wiley \& Sons, 2001

[11] Büschel, P. ; Tröltzsch, U. ; Kanoun, O.: Use of stochastic methods for robust parameter extraction from impedance spectra. In: Electrochimica Acta - (2011), - DOI 10.1016/j.electacta.2011.01.047

[12] Popkirov, G. S. ; Schindler, R. N.: A new impedance spectrometer for the investigation of electrochemical systems. In: Review of Scientific Instrument 63 (1992), No. 11, p. 5366-5372

[13] Tröltzsch, U. ; Kanoun, O. ; Tränkler, H.-R.: Characterizing aging effects of lithium ion batteries by impedance spectroscopy. In: Electrochimica Acta 51 (2006), No. 8-9, p. 1664-1672. - DOI 10.1016/j.electacta.2005.02.148

[14] Randles, J. E. B.: Kinetics of rapid electrode reactions. In: Discuss Faraday Soc. 1 (1947), p. 11-19

[15] Podlubny, I. ; Petras, I. ; Vinagre, B.M. ; O'Leary, P. ; Dorcak, L.: Analogue realizations of fractionalorder controllers. In: Nonlinear Dynamics 29 (2002), No. 1-4, p. 281-296. - DOI 10.1023/A:1016556604320

[16] Oustaloup, A. ; Levron, F. ; Mathieu, B. ; Nanot, F.M.: Frequency-band complex noninteger differentiator: Characterization and synthesis. In: IEEE Transactions on Circuits and Systems I: Fundamental Theory and Applications 47 (2000), No. 1, p. 25-39. - DOI 10.1109/81.817385 